\title{
On Generalized Inverses, Group Inverses And Reverse Order Law For Range Quaternion Hermitian Matrices (Q-Ep)
}

\author{
Dr.K.Gunasekaran ${ }^{1}$, S. Sridevi ${ }^{2}$, \\ ${ }^{I}$ Ramanujan Research centre, PG and Research Department of Mathematics, Government Arts College \\ (Autonomous), Kumbakonam - 612002 \\ ${ }^{2}$ Ramanujan Research centre, PG and Research Department of Mathematics, Government Arts College \\ (Autonomous), Kumbakonam - 612 002, Tamil Nadu, India.
}

Abstract: In this paper we discuss theGeneralized Inverses, Group Inverses And Reverse Order Law For Range Quaternion Hermitian Matrices (q-EP).

Keywords :Moore-Penrose inverse , $q$-EP matrix, Generalized Inverses for $q-E P$, Group Inverses for $q-E P$, Reverse Order Law for $q-E P$.

\section{Introduction}

Throught we shall deal with nxn quaternion matrices[7]. Let $\mathrm{A}^{*}$ denote the conjugate transpose of $\mathrm{A}$. Let $\mathrm{A}^{-}$be the generalized inverse of $\mathrm{A}$ satisfying $A A^{-} A$ and $\mathrm{z}$ be the MoorePenrose of $\mathrm{A}[6]$. Any matrix $A \in H_{n X n}$ is called $\mathrm{q}-\mathrm{EP}(2)$ if $\mathrm{R}(\mathrm{A})=\mathrm{R}\left(\mathrm{A}^{*}\right)$ and his called $\mathrm{q}-\mathrm{EP}_{\mathrm{r}}$, if $A$ is $q-E P$ and $r k(A)=r$, where $N(A), R(A)$ and $\operatorname{rk}(A)$ denote the null space, range space and rank of $A$ respectively. It is well known that sum and sum of parallel summable q-EP matrices are q-EP[3].In this paper we discuss theGeneralized Inverses, Group Inverses And Reverse Order Law For Range Quaternion Hermitian Matrices (q-EP).In this section, equivalent conditions for various generalized inverses of a $\mathrm{q}-\mathrm{EP}_{\mathrm{r}}$ matrix to be $\mathrm{q}-\mathrm{EP}_{\mathrm{r}}$ are determined. Generalized inverses belonging to the sets $\mathrm{A}\{1,2\}, \mathrm{A}\{1,2,3\}$ and $\mathrm{A}\{1,2,4\}$ of a $\mathrm{q}-\mathrm{EP}_{\mathrm{r}}$ matrix $\mathrm{A}$ are characterized.A generalized inverse $\mathrm{A} \in \mathrm{A}\{1,2\}$ is shown to be $\mathrm{q}-\mathrm{EP}_{\mathrm{r}}$ whenever $\mathrm{A}$ is $\mathrm{q}-\mathrm{EP}_{\mathrm{r}}$ under certain conditions in the following way.

\section{Theorem 1.1}

Let $\mathrm{A} \in \mathrm{H}_{\mathrm{nXn}}, \mathrm{X} \in \mathrm{A}\{1,2\}$ and $\mathrm{XA}, \mathrm{AX}$ are $\mathrm{q}-\mathrm{EP}_{\mathrm{r}}$ matrices. Then $\mathrm{A}$ is $\mathrm{q}-\mathrm{EP}_{\mathrm{r}} \Leftrightarrow \mathrm{X}$ is $\mathrm{q}-\mathrm{EP}_{\mathrm{r}}$

Proof

Since AX and XA are $\mathrm{q}-\mathrm{EP}_{\mathrm{r}}$, by theorem([2],11), we have $\mathrm{R}(\mathrm{AX})=\mathrm{R}\left((\mathrm{AX})^{*}\right)$ and $\mathrm{R}(\mathrm{XA})=\mathrm{R}\left(\left(\mathrm{XA}^{*}\right)\right.$. Since $X \in\{1,2\}$ we have $A X A=A, X A X=X$

Now,

$$
\begin{gathered}
\mathrm{R}(\mathrm{A})=\mathrm{R}(\mathrm{AX}) \\
=\mathrm{R}\left((\mathrm{AX})^{*}\right) \\
=\mathrm{R}\left(\mathrm{X}^{*} \mathrm{~A}^{*}\right) \\
=\mathrm{R}\left(\mathrm{X}^{*}\right) \\
\mathrm{R}\left(\mathrm{A}^{*}\right)=\mathrm{R}\left(\mathrm{A}^{*} \mathrm{X}^{*}\right) \\
=\mathrm{R}\left((\mathrm{XA})^{*}\right) \\
=\mathrm{R}(\mathrm{XA}) \\
=\mathrm{R}(\mathrm{X})
\end{gathered}
$$

Now, $A$ is $q-E P_{r} \Leftrightarrow R(A)=R\left(A^{*}\right)$ and $r k(A)=r$

$$
\begin{aligned}
& \Leftrightarrow R\left(X^{*}\right)=R(X) \text { and } \operatorname{rk}(A)=r k(X)=r \\
& \Leftrightarrow X \text { is q-EP } P_{r}
\end{aligned}
$$

Hence the theorem

\section{Remark 1.2}

In the above theorem, the conditions that both $\mathrm{AX}$ and $\mathrm{XA}$ to be $\mathrm{q}-\mathrm{EP}_{\mathrm{r}}$ are essential. For instance, let 


$$
\begin{aligned}
& \mathrm{A}=\left(\begin{array}{cc}
1 & k \\
-k & 1
\end{array}\right), \mathrm{A} \text { is } \mathrm{q}-\mathrm{EP}_{1} \\
& \mathrm{X}=\mathrm{A}=\left(\begin{array}{ll}
1 & 0 \\
0 & 0
\end{array}\right) \in \mathrm{A}\{1,2\} \\
& \mathrm{AX}=\left(\begin{array}{cc}
1 & 0 \\
-k & 0
\end{array}\right) \\
& \mathrm{XA}=\left(\begin{array}{ll}
1 & k \\
0 & 0
\end{array}\right)
\end{aligned}
$$

$\mathrm{AX}$ and $\mathrm{XA}$ are not $\mathrm{q}-\mathrm{EP}_{1}$. Also $\mathrm{X}$ is not $\mathrm{q}-\mathrm{EP}$

Now, we show that generalized inverses belonging to the sets $\mathrm{A}\{1,2,3\}$ and $\mathrm{A}\{1,2,4]$ of a $\mathrm{q}-\mathrm{EP}_{\mathrm{r}}$ matrix $\mathrm{A}$ is also $\mathrm{q}-\mathrm{EP}_{\mathrm{r}}$ under certain conditions in the following theorems.

\section{Theorem 1.3}

Let $A \in H_{n X n}, X \in A\{1,2,3\}, R(X)=R\left(A^{*}\right)$. Then $A$ is $q-E P_{r} \Leftrightarrow X$ is $q-E P_{r}$

\section{Proof}

Since $\mathrm{X} \in \mathrm{A}\{1,2,3\}$, we have $\mathrm{AXA}=\mathrm{A}, \mathrm{XAX}=\mathrm{X},(\mathrm{AX})^{*}=\mathrm{AX}$. Therefore,

$$
\begin{aligned}
& \mathrm{R}(\mathrm{A})=\mathrm{R}(\mathrm{AX}) \\
& =\mathrm{R}\left((\mathrm{AX})^{*}\right) \\
& =R\left(X^{*} A^{*}\right) \\
& =R\left(X^{*}\right) \\
& \mathrm{R}(\mathrm{X})=\mathrm{R}\left(\mathrm{A}^{*}\right) \Rightarrow \mathrm{XX}^{\dagger}=\mathrm{A}^{*}\left(\mathrm{~A}^{*}\right)^{\dagger}[\mathrm{by}[1]] \\
& \Rightarrow \mathrm{XX}^{\dagger}=\mathrm{A}^{*}\left(\mathrm{~A}^{\dagger}\right)^{*} \\
& \Rightarrow \mathrm{XX}^{\dagger}=\left(\mathrm{A}^{\dagger} \mathrm{A}\right)^{*} \\
& \Rightarrow \mathrm{XX}^{\dagger}=\mathrm{A}^{\dagger} \mathrm{A} \\
& \Rightarrow \mathrm{XX}^{\dagger}=\left(\mathrm{A}^{*}\right)\left((\mathrm{A})^{*}\right)^{\dagger} \\
& \Rightarrow \mathrm{X}=\mathrm{R}\left((\mathrm{A})^{*}\right) \\
& \Rightarrow \mathrm{R}(\mathrm{X})=\mathrm{R}\left(\mathrm{A}^{*}\right)
\end{aligned}
$$

Hence the theorem.

\section{Theorem 1.4}

Let $\mathrm{A} \in \mathrm{H}_{\mathrm{nxn}}, \mathrm{X} \in\{1,2,4\}, \mathrm{R}(\mathrm{A})=\mathrm{R}\left(\mathrm{X}^{*}\right)$. Then $\mathrm{A}$ is $\mathrm{q}-\mathrm{EP}_{\mathrm{r}} \Leftrightarrow \mathrm{X}$ is $\mathrm{q}-\mathrm{EP}_{\mathrm{r}}$

\section{Proof}

Since $\mathrm{X} \in \mathrm{A}\{1,2,4\}$, we have $\mathrm{AXA}=\mathrm{A}, \mathrm{XAX}=\mathrm{A},(\mathrm{XA}) *=\mathrm{XA}$.

Also.

$$
\begin{aligned}
\mathrm{R}(\mathrm{A}) & =\mathrm{R}\left(\mathrm{X}^{*}\right) . \text { Now } \\
\mathrm{R}\left(\mathrm{A}^{*}\right) & =\mathrm{R}\left(\mathrm{A}^{*} \mathrm{X}^{*}\right) \\
& =\mathrm{R}\left((\mathrm{XA})^{*}\right) \\
& =\mathrm{R}(\mathrm{XA}) \\
& =\mathrm{R}(\mathrm{X}) \\
\mathrm{A} \text { is } \mathrm{q}-\mathrm{EP}_{\mathrm{r}} \Leftrightarrow & \mathrm{R}(\mathrm{A})=\mathrm{R}\left(\mathrm{A}^{*}\right) \text { and } \mathrm{rk}(\mathrm{A})=\mathrm{r}
\end{aligned}
$$$$
\Leftrightarrow R\left(X^{*}\right)=R(X) \text { and } \operatorname{rk}(A)=\operatorname{rk}(X)=r \text { [by[2], 11] }
$$

\section{Remarks 1.5}

$$
\Leftrightarrow X \text { is } q-E P_{r}
$$

In particular, if $\mathrm{X}=\mathrm{A}^{\dagger}$ then $\mathrm{R}\left(\mathrm{A}^{\dagger}\right)=\mathrm{R}\left(A^{*}\right)$ holds. Hence $\mathrm{A}$ is $\mathrm{q}-\mathrm{EP}_{\mathrm{r}}$ is equivalent to $\mathrm{A}^{\dagger}$ is $\mathrm{q}-\mathrm{EP}_{\mathrm{r}}$. 


\section{Group Inverse of q-EP matrices}

In this section, the existence of the group inverse for q-EP matrices under certain condition is derived. It is well known that, for an EP matrix, group inverse exists and coincides with it Moore-Penrose inverse. However, this is not the case for a q-EP matrix.

For example,

$$
\text { Consider } \mathrm{A}=\left(\begin{array}{cc}
1 & i \\
-i & 1
\end{array}\right)
$$

$\mathrm{A}$ is q-EP, matrix, $\mathrm{A}^{2}=\left(\begin{array}{cc}2 & 2 i \\ -2 i & 2\end{array}\right), \operatorname{rk}(\mathrm{A})=\operatorname{rk}\left(\mathrm{A}^{2}\right)$

Therefore, by theorem [p.162[1]], group inverse $\mathrm{A}^{\dagger}$ does not exist for A. Here it is proved that for qEP matrix A, if the group inverse exists, it is also a q-EP matrix.

\section{Theorem 2.1}

Let $A \in H_{n x n}$ be $q-E P r$ and $r k(A)=r k\left(A^{2}\right)$. Then $A^{\#}$ exists and is $q-E P_{r}$

\section{Proof}

Since $\operatorname{rk}(A)=\operatorname{rk}\left(A^{2}\right)$, by theorem[p.162,[1] $A^{\#}$ exists for $A$. To show that $A^{\# i s ~} q-E P_{r}$, it is enough to show that

$R\left(A^{\#}\right)=R\left(\left(A^{\#}\right)^{*}\right)$

Since $A^{\#}=A^{\#} A$

$$
\begin{aligned}
& \text { We have } \mathrm{R}(\mathrm{A})=\mathrm{R}\left(A A^{\#}\right) \\
& =\mathrm{R}\left(A^{\neq} A\right) \\
& =\mathrm{R}\left(A^{\neq}\right) \\
& A A^{\neq} A=A \Rightarrow A^{*}=A^{*}\left(A^{\neq}\right)^{*} A^{*} \\
& \text { Therefore } \mathrm{R}\left(A^{*}\right)=\mathrm{R}\left(A^{*}\left(A^{\neq}\right)^{*} A^{*}\right) \\
& =\mathrm{R}\left(A^{*}\left(A^{\neq}\right)^{*}\right) \\
& =\mathrm{R}\left(\left(A^{\neq} A\right)^{*}\right) \\
& =\mathrm{R}\left(\left(A A^{\#}\right)^{*}\right) \\
& =\mathrm{R}\left(\left(A^{\neq}\right)^{*} A^{*}\right) \\
& =\mathrm{R}\left(\left(A^{\mp}\right)^{*}\right)
\end{aligned}
$$

Now,

$$
\begin{gathered}
\mathrm{A} \text { is } \mathrm{q}-\mathrm{EP}_{\mathrm{r}} \Rightarrow \mathrm{R}(\mathrm{A})=\mathrm{R}\left(A^{*}\right) \text { and } \mathrm{rk}(\mathrm{A})=\mathrm{r} \\
\Rightarrow \mathrm{R}\left(A^{*}\right)=\mathrm{R}\left(\left(A^{\neq}\right)^{*}\right) \text { and } \\
\quad \operatorname{Rk}(\mathrm{A})=\operatorname{rk}\left(A^{\neq}\right)=\mathrm{r} \\
\Rightarrow A^{\neq} \text {is } \mathrm{q}-\mathrm{EP}_{\mathrm{r}}
\end{gathered}
$$

Hence the Theorem.

\section{Remark 2.2}

In the above theorem the condition that $\operatorname{rk}(\mathrm{A})=\mathrm{rk}\left(\mathrm{A}^{2}\right)$ is essential. Therefore, $A^{\neq}$does not exist for a q-EP matrix A. Thus, for a q-EP matrix A, if $A^{\neq}$exists then it is also q-EPr.

\section{Theorem 2.3}

For at $\mathrm{H}_{\mathrm{nxn}}$, if $A^{\neq}$exists then, $\mathrm{A}$ is $\mathrm{q}-\mathrm{EP} \Leftrightarrow A^{\neq}=\mathrm{A}^{\dagger}$

Proof

$\mathrm{A}$ is $\mathrm{q}-\mathrm{EP} \Leftrightarrow \mathrm{A}$ is Ep $\quad$ [By Theorem11,[2]]

$$
\Leftrightarrow A^{\neq}=\mathrm{A}^{\dagger} \text { [p.164[8]] }
$$

Hence the theorem.

\section{Theorem 2.5}

For $\mathrm{A} \in \mathrm{H}_{\mathrm{nxn}}$, A is q-EPr $\Leftrightarrow \mathrm{A}^{\dagger}=$ polynomial in A 
Proof

It is clear that if $\mathrm{A}^{\dagger}=\mathrm{f}(\mathrm{A})$ for some polynomial $\mathrm{f}(\mathrm{X})$, then $\mathrm{A}$ commutes with (A) ${ }^{\dagger}$ for some polynomial $\mathrm{f}(\mathrm{X})$, then A commutes with (A) ${ }^{\dagger}$

$\Rightarrow \mathrm{A}$ is q-EP

$$
\Rightarrow \mathrm{AA}^{\dagger}=\mathrm{A}^{\dagger} \mathrm{A}
$$

Conversely,

Let $\mathrm{A}$ be $\mathrm{q}-\mathrm{EP}_{\mathrm{r}}$, then $\mathrm{AA}^{\dagger}=\mathrm{A}^{\dagger} \mathrm{A}$ and $\mathrm{A}^{\dagger} \mathrm{A}=\mathrm{AA}^{\dagger}$.

Now, we will prove the $\mathrm{A}^{\dagger}$ can be expressed as polynomial in $\mathrm{A}$.

$$
\text { Let }(\mathrm{A})^{\mathrm{s}}+\lambda_{1}(\mathrm{~A})^{\mathrm{s}+1}+\lambda_{2}(\mathrm{~A})^{\mathrm{s}+2}+\ldots \ldots \ldots+\lambda_{\mathrm{q}}(\mathrm{A})^{\mathrm{s}+\mathrm{q}}=0
$$

$\mathrm{Be}$ the minimum polynomial of $\mathrm{A}$. Then $\mathrm{s}=0$ or $\mathrm{s}=1$.

For suppose that $\mathrm{s} \geq 2$, then

$$
\begin{aligned}
& \mathrm{A}^{\dagger}\left[(\mathrm{A})^{\mathrm{s}}+\lambda_{1}(\mathrm{~A})^{\mathrm{s}+1}+\ldots \ldots \ldots . .+\lambda_{\mathrm{q}}(\mathrm{A})^{\mathrm{s}+\mathrm{q}}\right]=0, \\
& {\left[\mathrm{AA}^{\dagger} \mathrm{A}\right] \mathrm{A}^{\mathrm{s}-2}+\lambda_{1}\left[\mathrm{AA}{ }^{\dagger} \mathrm{A}\right] \mathrm{A}^{\mathrm{s}-1}+\ldots \ldots \ldots .+\lambda_{9}\left[\mathrm{AA}{ }^{\dagger} \mathrm{A}\right] \mathrm{A}^{\mathrm{s}+\mathrm{q}-2}=0} \\
& \text { That is }(\mathrm{A})^{\mathrm{s}-1}+\lambda_{1}(\mathrm{~A})^{\mathrm{s}}+\ldots \ldots \ldots . .{ }_{9}(\mathrm{~A})^{\mathrm{s}+\mathrm{q}-1}=0 \\
& \text { Which is contradiction. } \\
& \text { If } \mathrm{s}=0 \text { then }
\end{aligned}
$$

$$
\begin{aligned}
& \left(\mathrm{A}^{\dagger}\right)=\mathrm{A}^{-1}=-\lambda_{1} \mathrm{I}-\lambda_{2}(\mathrm{~A})-\ldots \ldots \ldots \ldots \ldots \ldots . . . \lambda_{\mathrm{q}}(\mathrm{A})^{\mathrm{q}-1}
\end{aligned}
$$

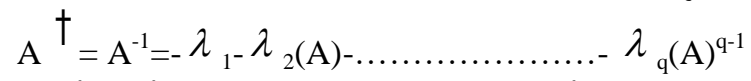

$$
\begin{aligned}
& =\left[-\lambda_{1} \mathrm{I}-\lambda_{2} \mathrm{~A}-\ldots \ldots \ldots \ldots \ldots \ldots \ldots \ldots . . . \cdots \ldots{ }_{\mathrm{q}}(\mathrm{A})^{\mathrm{q}-1}\right]
\end{aligned}
$$

$\mathrm{A}^{\dagger}=$ polynomial in $\mathrm{A}$

$$
\begin{aligned}
& \text { If } \mathrm{s}=1 \text {, then }\left(\mathrm{A}^{\dagger}\right)\left[\mathrm{A}+\lambda_{1}(\mathrm{~A})^{2}+\ldots \ldots+\lambda_{\mathrm{q}}(\mathrm{A})^{\mathrm{q}+1}\right]=0 \\
& \text { and it follows that } \\
& \mathrm{A}^{\dagger} \mathrm{A}=-\lambda_{1}(\mathrm{~A})-\lambda_{2}\left(\mathrm{~A}^{2}\right)-\ldots \ldots \ldots \ldots . . . \lambda_{\mathrm{q}(\mathrm{A})} \mathrm{q}^{\mathrm{q}} \text { is a polynomial in } \mathrm{A} \text {. } \\
& \mathrm{A}^{\dagger}=\left[\mathrm{A}^{\dagger} \mathrm{A}\right] \mathrm{A}{ }^{\dagger} \\
& =-\lambda_{1(\mathrm{~A})}^{\dagger}(\mathrm{A})-\lambda_{2}(\mathrm{~A})- \\
& =\left[-\lambda_{1} \mathrm{I}_{-} \lambda_{2}(\mathrm{~A})-\ldots \ldots \ldots \ldots \ldots . .-\lambda_{\mathrm{q}}(\mathrm{A})^{\mathrm{q}-1}\right] \\
& \mathrm{A}^{\dagger}=\text { polynomial in } \mathrm{A} \text {. }
\end{aligned}
$$

However,

Hence the theorem.

\section{Reverse order law for q-EP matrices}

For any two non singular matrices $A, B \in H_{n \times n}(A B)^{-1}=B^{-1} A^{-1}$ holds. However, it is not true for generalized inverses of matrices [15]. In general, (AR) $\dagger \neq \mathrm{B}^{\dagger} \mathrm{A}^{\dagger}$ for any two matricesa and B. we say that reverse order law holds for Moore-Penrose inverse of the product of $A$ and $B$, if $(A B){ }^{\dagger}=B^{\dagger}{ }^{\dagger}$. It is well known that [P.181,[1]], (AB) ${ }^{\dagger}=\mathrm{B}^{\dagger} \mathrm{A}^{\dagger}$ if and only if $\mathrm{R}\left(\mathrm{BB}^{*} \mathrm{~A}\right) \subseteq \mathrm{R}\left(\mathrm{A}^{*}\right)$ and $\mathrm{R}\left(\mathrm{A}^{*} \mathrm{AB}\right) \subseteq \mathrm{R}(\mathrm{B})$.In this section, for a pair of q-EPr matrices $A$ and $B$, necessary and sufficient condition for $(\mathrm{AB}){ }^{\dagger}=\mathrm{B}^{\dagger_{\mathrm{A}}} \dagger_{\text {given. }}$

\section{Theorem}

If $\mathrm{A}$ and $\mathrm{B}$ are $\mathrm{q}-\mathrm{EPr}$ matrices with $\mathrm{R}(\mathrm{A})=\mathrm{R}\left(\mathrm{B}^{*}\right)$ then $(\mathrm{AB}){ }^{\dagger}=\mathrm{B}^{\dagger} \mathrm{A}^{\dagger}$

Proof

Since A is q-EPr,

$$
\begin{aligned}
& \Rightarrow R(A)=R\left(A^{*}\right) \\
& \Rightarrow R\left(B^{*}\right)=R(A) \quad \text { (B is q-EPr) }
\end{aligned}
$$




$$
\begin{aligned}
& \Rightarrow \mathrm{R}(\mathrm{B})=\mathrm{R}\left(\mathrm{A}^{*}\right) \\
& \Rightarrow \mathrm{R}(\mathrm{B})=\mathrm{R}\left(\mathrm{A}^{\dagger}\right)
\end{aligned}
$$

That is, given $\mathrm{x} \in \mathrm{C}_{\mathrm{nxn}}$, there exists $\mathrm{y} \in \mathrm{C}_{\mathrm{n}}$ such that $\mathrm{Bx}=\mathrm{A}^{\dagger} \mathrm{y}$

$$
\text { Now, } \begin{aligned}
\mathrm{Bx}_{\mathrm{A}} \mathrm{A}^{\dagger} \mathrm{y} & \Rightarrow\left(\mathrm{B}^{\dagger} \mathrm{A}^{\dagger} \mathrm{A}\right) \mathrm{Bx}=\left(\mathrm{B}^{\dagger} \mathrm{A}^{\dagger} \mathrm{A}\right) \mathrm{A}^{\dagger} \mathrm{y} \\
& \Rightarrow \mathrm{B}^{\dagger} \mathrm{A}^{\dagger} \mathrm{ABx}=\mathrm{B}^{\dagger} \mathrm{A}^{\dagger} \mathrm{AA}^{\dagger} \mathrm{y} \\
& \Rightarrow \mathrm{B}^{\dagger} \mathrm{A}^{\dagger} \mathrm{ABx}=\mathrm{B}^{\dagger} \mathrm{A}^{\dagger} \mathrm{y} \\
& \Rightarrow \mathrm{B}^{\dagger} \mathrm{A}^{\dagger} \mathrm{ABx}=\mathrm{B}^{\dagger} \mathrm{Bx}
\end{aligned}
$$

Similarly,

Since $\mathrm{B}^{\dagger} \mathrm{B}$ is hermitian, it follows that $\mathrm{B}^{\dagger} \mathrm{A}^{\dagger} \mathrm{AB}$ is hermitian.

$$
\begin{aligned}
\mathrm{A}^{\dagger} \mathrm{y}=\mathrm{Bx} & \Rightarrow\left(\mathrm{ABB}^{\dagger}\right) \mathrm{A}^{\dagger} \mathrm{y}=\left(\mathrm{ABB}^{\dagger} \mathrm{B}\right) \mathrm{x} \\
& \Rightarrow \mathrm{ABB}^{\dagger} \mathrm{A}^{\dagger} \mathrm{y}=\mathrm{A}\left(\mathrm{BB}^{\dagger} \mathrm{B}\right) \mathrm{x} \\
& \Rightarrow \mathrm{ABB}^{\dagger} \mathrm{A}^{\dagger} \mathrm{y}=\mathrm{A}(\mathrm{Bx}) \\
& \Rightarrow \mathrm{ABB}^{\dagger} \mathrm{A}^{\dagger} \quad \mathrm{y}=\mathrm{A}_{\left(\mathrm{A}^{\dagger} \mathrm{y}\right)} \\
& \Rightarrow \mathrm{ABB}^{\dagger} \mathrm{A}^{\dagger} \mathrm{y}=\mathrm{AA}^{\dagger} \mathrm{y}
\end{aligned}
$$

Since $\mathrm{AA}^{\dagger}$ is hermitian, it follows that $\mathrm{ABB}^{\dagger} \mathrm{A}^{\dagger}$ is hermitian. Further, by theorem [8]

$$
\begin{aligned}
& \mathrm{R}(\mathrm{A})=\mathrm{R}(\mathrm{B}) \Rightarrow \mathrm{AA}^{\dagger}=\mathrm{BB}^{\dagger} \\
& \mathrm{R}\left(\mathrm{A}^{\dagger}\right)=\mathrm{R}(\mathrm{B}) \Rightarrow \mathrm{A}^{\dagger}\left(\mathrm{A}^{\dagger}\right)^{\dagger}=\mathrm{BB}^{\dagger} \\
& \Rightarrow \mathrm{A}^{\dagger} \mathrm{A}=\mathrm{BB}^{\dagger}
\end{aligned}
$$

Hence (AB) $\left(\mathrm{B}^{\dagger} \mathrm{A}^{\dagger}\right)(\mathrm{AB})=\mathrm{ABB}^{\dagger}\left(\mathrm{A}^{\dagger} \mathrm{A}\right) \mathrm{B}$

$$
\begin{aligned}
& =\mathrm{ABB}^{\dagger}\left(\mathrm{B}^{\dagger} \mathrm{BB}^{\dagger}\right) \mathrm{B} \\
& =(\mathrm{AB})\left(\mathrm{B}^{\dagger}\right) \mathrm{B} \\
& =\mathrm{A}\left(\mathrm{BB}^{\dagger} \mathrm{B}\right) \\
& =\mathrm{A}(\mathrm{B}) \\
& =\mathrm{AB}
\end{aligned}
$$

$111^{\text {ly }}\left(\mathrm{B}^{\dagger} \mathrm{A}^{\dagger}\right)(\mathrm{AB})\left(\mathrm{B}^{\dagger} \mathrm{A}^{\dagger}\right)=\mathrm{B}^{\dagger} \mathrm{A}^{\dagger}$.

Thus, $\mathrm{B}^{\dagger} \mathrm{A}^{\dagger}$ satisfies the definition of the Moore-Penrose inverse, that is (AB) ${ }^{\dagger}=\mathrm{B}^{\dagger} \mathrm{A}^{\dagger}$ Hence the theorem.

\section{Reference}

[1]. Ben Isreal. A and Greville. TNE : Generalized Inverses, Theory andapplications ; Wiley and Sons , New York( 1974).

[2]. KatzT.J and Pearl M.H: on Ep $\mathrm{p}_{\mathrm{r}}$ and Normal Ep $\mathrm{p}_{\mathrm{r}}$ matriceJ.res.Nat.Bur.Stds. 70B, 47-77(1966).

[3]. Gunasekaran.K and Sridevi.S: On Range Quaternion Hermittian MatricesInter;J;Math.,Archieve-618, 159-163

[4]. Gunasekaran.G and Sridevi.S: On Sums of Range Quaternion Hermittian Matrices; Inter;J.Modern Engineering Res-.5, ISS.11. 44 49(2015)

[5]. Marsagila.GandStyanG.P.H :Equalities and Inequalities for rank of Matrices;lin.Alg.Appl.,2,269-292(1974)

[6]. Rao.CR and Mitra.SK: Generalized inverse of matrices and its Application: Wiley and Sons, Newyork(1971)

[7]. [Zhang.F, Quaternions and matrices of quaternions, linear Algebra and its Application, 251 (1997), 21 - 57 